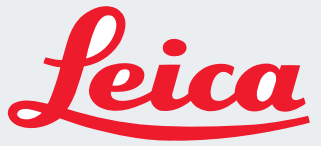

M I C ROS Y STEM S

\title{
Dynamic imaging sheds light on embryonic development processes
}

\author{
A complete software-controlled system developed by Leica Microsystems opens up new paths in \\ development research. The motorized fluorescence stereomicroscope, digital camera and fluorescence \\ imaging software work together with precision, easily handling even complex time-lapse and Z-stack \\ experiments with quick filter change routines and deconvolution.
}

Without dynamic imaging methods, capturing embryonic development processes would be unthinkable. To automate laborious timelapse experiments on living zebrafish embryos, developmental biologists at the National Research Center for Environment and Health (GSF), in Neuherberg, Germany, tested a complete software-controlled system developed by Leica Microsystems. The motorized fluorescence stereomicroscope, fluorescence imaging software and digital camera work together with precision, easily handling even complex time-lapse and Z-stack experiments with quick filter change routines and deconvolution. After comprehensive all-around testing, as well as specific tests to determine the precision of the interaction between hardware and software, it became obvious that, in every respect, the Leica system enriches everyday scientific work in a zebrafish neuroimaging laboratory, and offers a cost-efficient alternative to expensive instruments for intravital microscopic imaging.

The complete Leica system— put to the test with zebrafish Researchers from the Zebrafish Neuroimaging Group at the GSF use the zebrafish as a model organism for intravital studies of genetic development. Native to India's Ganges River, this popular research subject is highly sought after in biology labs around the world. A look through the microscope reveals the secret of its popularity: the zebrafish embryo is transparent, making it possible to observe first-hand the development of organs such as the eye as the organism grows. By injecting contrast agents such as fluorescein isothiocyanate (FITC), or DNA or RNA molecules encoding fluorescent proteins, it becomes possible to visualize subcellular structures like nuclei, mitochondria or microfilaments using fluorescence microscopy.

\section{Ingrid Haack}

Stereo \& Macroscope Systems, Leica Microsystems (Schweiz) AG, CH-9435 Heerbrugg Switzerland

e-mail:smspromotion@leica-microsystems.com

PUBUISHED ONLINE 21 ○CTOBER 2005; DQ:10.1038/ NMETH810

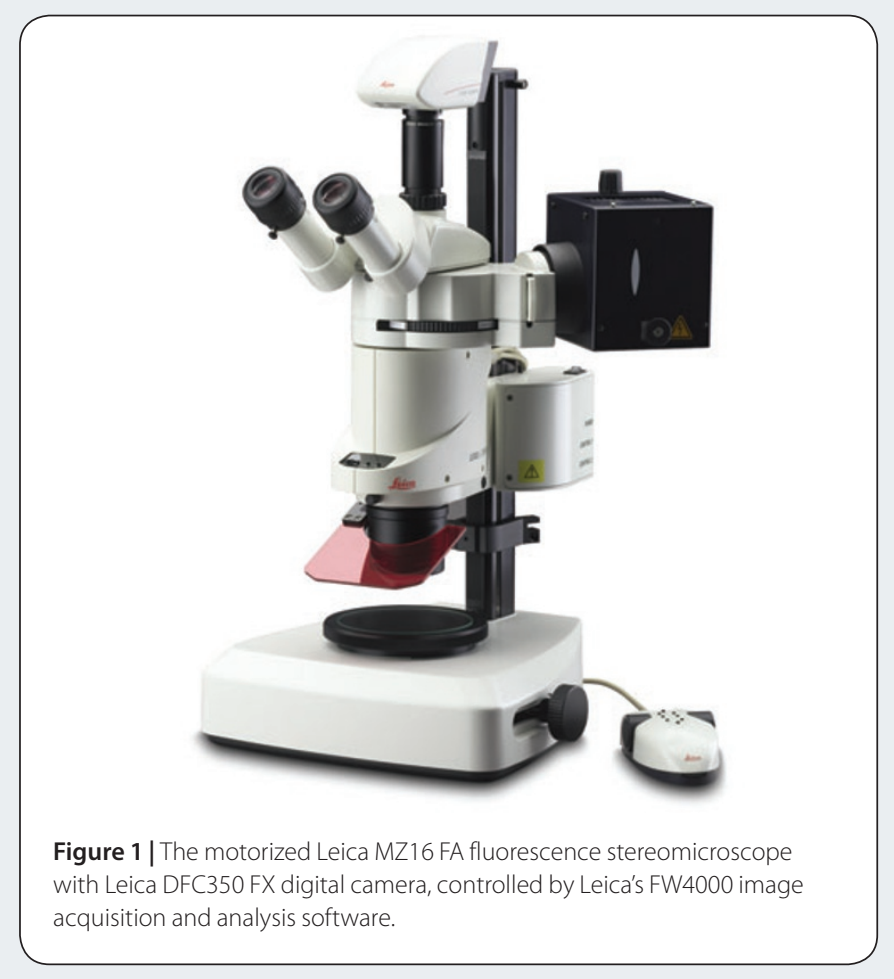

Some experiments, however, such as those involving fast filter exchange routines for imaging double-labeled moving cells, are unthinkable without a motorized microscope operated by software. The GSF researchers tested a software-controlled motorized dissecting microscope from Leica Microsystems in various experiments on living zebrafish embryos, to evaluate its usefulness in biological imaging tasks of living organisms. Leica's FW4000 image acquisition and analysis software supports Leica DFC fluorescence cameras and the Leica MZ16 FA fluorescence stereomicroscope (Fig. 1), offering users a complete imaging solution that can be tailored to their application requirements and budget. This software controls all motorized parts of the MZ16 FA, such as the fully apochromatic 16:1 


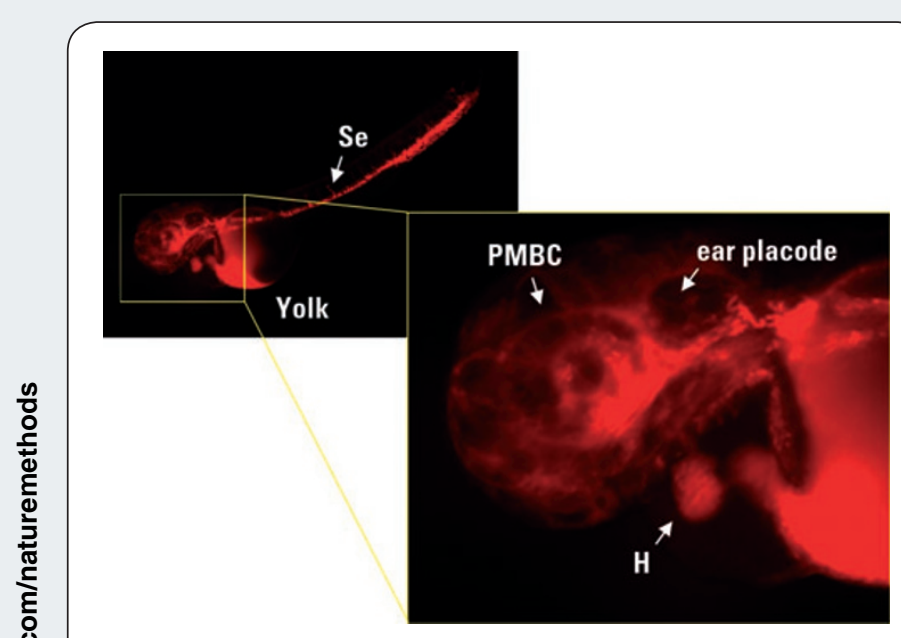

Figure $\mathbf{2}$ | Time-lapse images of a zebrafish embryo with injected contrast medium.

zoom, the focusing drive, the filter wheel and the UV shutter, and allows fully automated time-lapse and Z-stack recording as well as processing of the obtained images. In addition, the MZ16 FA meets all requirements for perfect image support. The MZ16 FA offers the largest zoom range of all motorized fluorescence stereomicroscopes, featuring magnifications of up to 920x; the highest resolution, from $840 \mathrm{lp} / \mathrm{mm}$ up to $1,500 \mathrm{lp} / \mathrm{mm}$; and the finest visible structure width, from $0.3 \mu \mathrm{m}$ up to $0.6 \mu \mathrm{m}$.

\section{Complex experiments with time-lapse, filter change, Z-} stack and successful deconvolution

The FW4000 software was able to reliably automate all time-lapse experiments in bright-field and fluorescence modes, and could handle the imaging of rapidly occurring processes, such as the various phases of the heartbeat (Fig. 2). Even complex time-lapse experiments using three fluorescence filters functioned perfectly. UV was used to excite three different pigments, GFP3, DAPI and Texas red, within the cells of a zebrafish embryo in rapid succession; the automated filter change process was fast and vibration-free, resulting in satisfying images without pixel shifts.

The motorized focusing drive on the Leica MZ16 FA allows automated imaging in various planes along the $z$ axis. As a test, a $Z$ stack in 15 planes was taken of a double-labeled zebrafish embryo (Fig. 3). With the FW4000 software, the focal displacements and the change routine for the two fluorescence filters functioned reliably and without vibration. The images from the individual channels (GFP3 and Texas red) for each Z plane were then composed into a single image using the FW4000 software (Fig. 3c). This can produce a

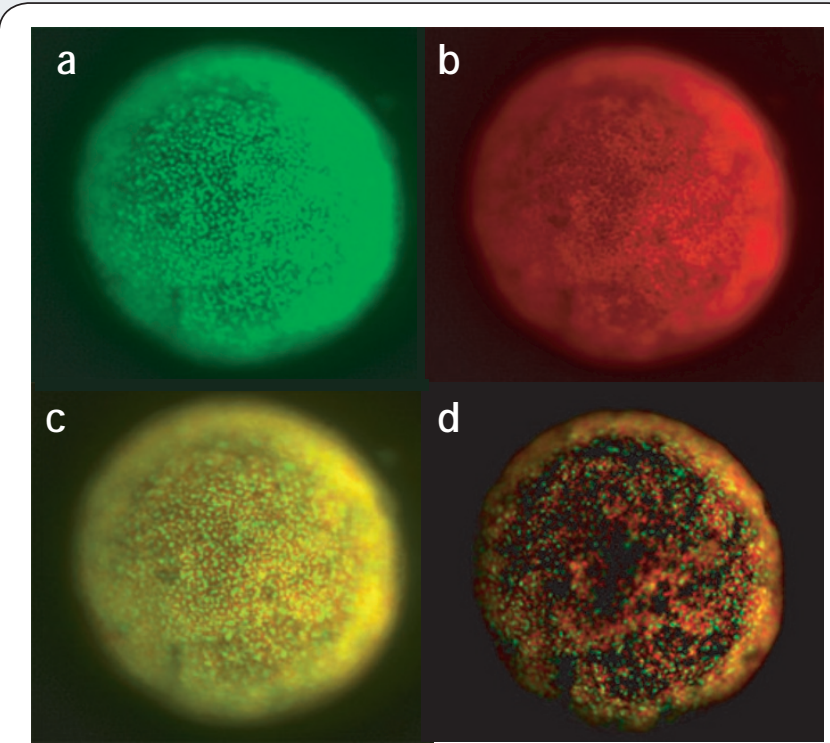

Figure 3 | Zebrafish embryo with multicolored fluorescence-marked nuclei and cellular membranes; level 8 of the Z stack. (a) GFP3 channel with fluorescence-marked green cell nuclei. (b) Texas Red channel, with fluorescence-marked red cellular membranes. (c) Composite of $\mathbf{a}$ and b, before deconvolution. (d) After deconvolution, yellow areas have disappeared almost completely (that is, the fluorescence signals are correctly assigned to their focal plane).

blurry image, as is the case here, because regular images taken with a stereomicroscope contain a lot of information from areas outside of the focal plane. Also, the overlapping 'red' and 'green' images result in a yellow color that misleadingly indicates colocalization of nuclei and cellular membranes. The Leica Deblur imaging software provides the solution to this problem with its deconvolution function, which assigns nonfocused light signals to their original focal plane. The deconvolution worked very well; not only was the composite sharper (Fig. 3d), but the subcellular structures also appeared in convincingly high resolution, and the yellow areas disappeared almost completely.

\section{Convincing results}

The FW4000 software package from Leica Microsystems combines a variety of powerful features, yet is quite easy to learn and to use, and offers a very promising tool for automating time-lapse and Z-stack experiments. In combination with the Leica MZ16 FA motorized fluorescence stereomicroscope, the FW4000 package can open up new paths in development research.

This article was submitted to Nature Methods by a commercial organization and has not been peer reviewed. Nature Methods takes no responsibility for the accuracy or otherwise of the information provided. 